\title{
Contribution à l'étude des Microphallidae Travassos, 1920 (Trematoda)
}

XXIII. Les espèces décrites au Brésil par L. Travassos

\author{
par S. DEBLOCK \\ Laboratoire de Parasitologie, Faculté de Pharmacie, \\ rue Laguesse, F. 59 - Lille
}

\begin{abstract}
Résumé
Après avoir consulté les préparations conservées à l'Institut Oswaldo-Cruz, l'auteur apporte des compléments d'information concernant Maritrema pulcherrima Travassos, 1929; Odhneria odhneri Trav., 1921; Levinseniella jaegerskioeldi Trav., 1920 qui paraît synonyme de Gynaecotyla adunca (Linton, 1905) ; Levinseniella cruzi Trav., 1920; Maritrema nicolli Trav., 1920 qui n'appartient pas au genre Maritrema; et enfin Apophallus similinus Trav., 1920 qui est du genre Microphallus Ward.
\end{abstract}

\section{Summary}

After studying the specimens which are preserved at the Oswaldo-Cruz Institute, the author brings complementary information concerning Maritrema pulcherrima Travassos, 1929 ; Odhneria odhneri Trav., 1921; Levinseniella jaegerskioeldi Trav., 1920 which seems synonymous of Gynaecotyla adunca (Linton, 1905); Levinseniella cruzi Trav., 1920; Maritrema nicolli Trav., 1920 which does not belong to the genus Maritrema, and at last Apophallus simillimus Trav., 1920 which belongs to the genus Microphallus Ward. 


\section{INTRODUCTION}

Plusieurs espèces de Microphallidés décrites par Lauro Travassos constituent à des titres divers (imprécision des descriptions et de l'iconographie originales, interprétation discutable de certaines structures anatomiques) des énigmes du point de vue helminthologique. Pour essayer de les résoudre, nous avons obtenu, en nous recommandant de notre ami le professeur A.-G. Chabaud du Muséum d'Histoire Naturelle de Paris - et grâce à l'obligeance de $\mathbf{M}^{\mathrm{me}}$ Anna Kohn-Hoineff - quelques-unes des préparations originales de l'auteur sud-américain conservées dans les collections de l'Institut Oswaldo Cruz de Rio de Janeiro (Brésil). Ces préparations, toutes colorées au carmin et montées au baume, ont été examinées au microscope à lumière transmise ordinaire ou modifiée soit par le contraste de phase, soit par le contraste interférentiel. Certains exemplaires, apparemment uniques, montés depuis soixante ans ont subi un processus regrettable de « vitrification »; aussi ne montrent-ils pas tous les détails anatomiques indispensables à une identification précise. Les paragraphes qui suivent font le point des observations possibles que nous avons faites.

\section{I. - MARITREMATINAE}

\section{$1^{\circ}$ Maritrema pulcherrima Travassos, 1929}

PrÉPARATION n ${ }^{\circ} 6.197$.

Hôte: Didelphis aurita Wied (Mammifère Marsupial).

НАвітAт : Intestin grôle.

Localisation géographiQue: Angra dos Reis (Rio).

DATE DE RÉCOLTE : 1.7.1927.

MATÉRIEL OBSERVÉ: La préparation contient une trentaine d'exemplaires du parasite, très aplatis à la fixation, de morphologie très homogène, et en bon état de conservation (fig. 1).

Les caractéristiques principales de l'espèce, bien décrite par l'auteur, sont résumables ainsi :

Corps trapu dont la partie antérieure pré-caecale est très courte à l'état naturel.

Forte disparité ventousaire (V.O. > V.V.).

Esophage très court, de longueur voisine de celle du pharynx.

Caeca courts très divergents ne dépassant pas le niveau de la ventouse ventrale.

Ovaire assez nettement lobé, dextre ou sub-médian dextre, partiellement engagé sous la ventouse ventrale.

Utérus disposé en arrière de l'acétabulum et des testicules qui ne sont pas recouverts. Absence d'anses utérines extra-testiculaires. 


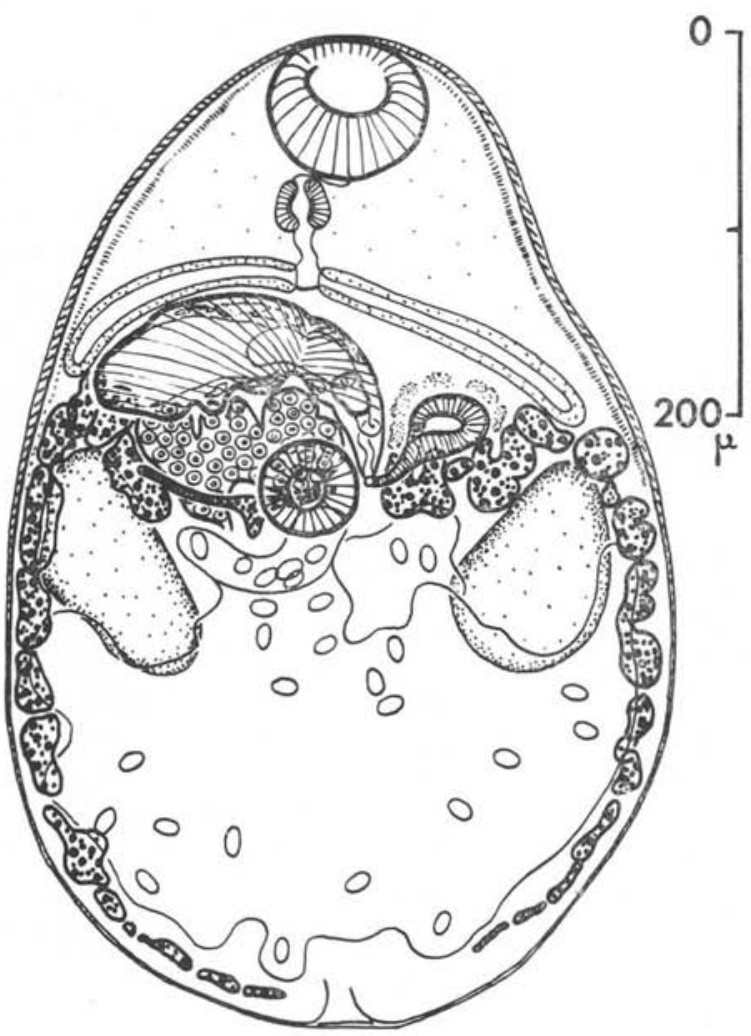

FIG. 1. - Maritrema pulchsrrima Trav., 1928. Syntype. Vue ventrale

Eufs abondants. Métraterme très bien différencié sous la forme d'un tube musculeux de 80-100 × 12-13 $\mu$ de diamètre, à parois de 2,5 $\mu$ d'épaisseur. Vitellogènes disposés selon les exemplairzz en anneau sub-complet ou en fər à cheval, et formés de follicules généralement nettement plus volumineux en avant des testicules qu'en arrière ; plusieurs exemplaires montrent en outre une file de follicules récurrents en avant des testicules, parfois relativement longue $(40$ à $50 \mu)$ et franchissant latéralement le fond des caeca, d'un côté ou de l'autre du distome.

Testicules largement séparés. Poche du cirre délimitée par une paroi musculeuse épaisse de $2 \mu$ environ, formée de fibres hélicoïdales bien individualisées sur tous les exemplaires. Ces fibres présentent la particularité originale de se disposer en chevron; l'axe du chevron est disposé selon l'axe longitudinal du corps, juste en avant de la ventouse ventrale et son emplacement est souvent le siège d'une légère striction du diamètre transversal de la poche du cirre ; la pointe du chevron est dirigée vers l'extrémité posté- 
rieure du distome du côté ventral et vers l'extrémité antérieure du côté dorsal. La poche du cirre contient une vésicule séminale sacculaire volumineuse qui emplit les $2 / 3$ postérieurs ; il lui fait suite successivement un très court canal $(10 \mu)$, une dilatation ampullaire de $17 \mu$ de diamètre environ représentant probablement la pars prostatica et enfin, le canal du cirre invaginé, long de 30 à $35 \mu \times 7 \mu$ de $\varnothing$ environ, à parois minces et glabres. Aucun exemplaire ne présente le cirre évaginé. La glande prostatique est bien visible, en manchon sur la périphérie des $2 / 3$ distaux de la poche du cirre.

Vésicule excrétrice et solénocytes non visibles.

Les détails observés permettent de compléter comme suit le paragraphe de la clé de détermination des espèzes du genre Maritrema (voir Deblock, 1972. Paragraphe I-2, 1, 4 , report $\left.n^{\circ} 37\right)$.

«Corps de taille moyenne 400-500 $\mu$ (à l'état très comprimé), trapu. V.O. de 80-100 $\mu$. V.V. de 40-48 $\mu$ pré-équatoriale. V.O./V.V. $=2 / 1$. Pharynx de 25-30 $\mu$. Esophage très bref de 10 à $20 \mu$. Caeca de 110-160 $\mu$ pré-sacculaires et pré-acétabulaires. Poche du cirre transversale de 120-190 $\mu$ de long à parois épaissies formées de fibres musculaires apparentes à disposition hélicoïdale et figurant un chevron pointe en bas sur la face ventrale et pointe en haut sur la face dorsale de la poche. Cirre glabre. Ovaire lobé sub-médian dextre. Anses utérines n'entourant pas la totalité des testicules. Métraterme musculeux long de 80-100 $\mu$ à paroi de $2 \mu$ d'épaisseur. Eufs de 16-18 $\mu$. Anneau vitellin complet ou en fer-à-cheval.

Parasite du tube digestif d'un mammifère (Marsupial) au Brésil $\ldots \ldots \ldots \ldots \ldots \ldots \ldots$ M. pulcherrima Travassos, 1929. »

\section{$2^{\circ}$ 《Maritrema 》 (?) nicolli}

PRÉPARATION n ${ }^{\circ} 2.079$.

Hôte : Daphila bahamensis (L.) (Oiseau Ansériforme).

HaвiтAт : Tube digestif.

LOCALISATION GÉOgRAPHIQUe : Manguinhos (Rio).

DATE DE RÉCOLTE : 5.12.1917.

MATÉRIEL OBSERVÉ : La préparation contient un unique exemplaire de l'espèce ; il a subi le processus de la « vitrification ». Très peu de détails restent visibles. Il correspond vraisemblablement à l'exemplaire figuré dans la planche XXV de l'auteur.

L'orientation du distome dans la préparation ne peut être déterminée que par la position de l'orifice de la ventouse orale : le tube digestif, l'acétabulum, le pore génital, le contenu de la poche du cirre de $115 \times 28 \mu$ et le volumineux complexe atrio-métratermique de $40 \times 50 \mu$ situé en avant du testicule gauche sont totalement indéchiffrables. (Cf. fig. 2). 
On ne peut préciser que les points suivants : cuticule totalement épineuse, depuis le niveau de la ventouse orale jusqu'à celui du pore excréteur, sans modification notable de la taille des épines $(4 \mu)$. Ventouse orale non régulièrement circulaire mais d'un contour inédit, de $30 \times 32 \mu$. Ovaire lobé sub-médian dextre, de $30 \mu$ de $\varnothing$. Testicules symétri-

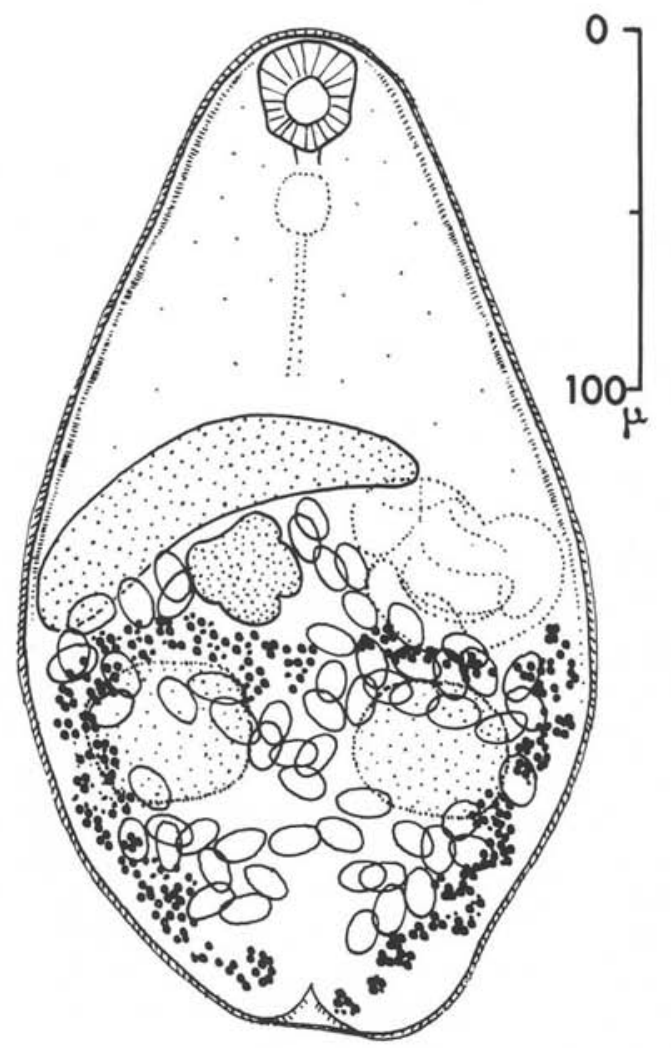

FIG. 2. - Maritrema nicolli Trav., 1920. Holotype. Vue ventrale. Les pointillés correspondent à des contours d'organes à peine élucidables

ques post-ovariens. Vitellogènes en anneau complet périphérique, latéro-ventraux, comme dans le genre Maritrema Nicoll. Anses utérines entourant les testicules et l'ovaire et remontant antérieurement jusqu'à la poche du cirre ; nombre d'œufs relativement réduit, de 15 à $16 \mu$ de long.

Discussion. - Ces caractères trop fragmentaires ne permettent cependant pas de confirmer l'appartenance de l'espèce nicolli Travassos, 1920 au genre Maritrema, ni 
même d'une façon certaine à la famille des Microphallidés. Néanmoins, la volumineuse formation atriale pourrait faire évoquer un genre de transition, analogue aux genres Sphairiotrema Deblock et Tran Van Ky, 1966 ou Androcotyla Deblock et Heard, 1970, mais que la position des vitellogènes apparenterait davantage aux Maritrematinés qu'aux Microphallinés.

\section{$3^{\circ}$ Odbneria odbneri Travassos, 1921}

PrÉPARATION $\mathrm{n}^{\circ} 2.085$.

Hôte : Nyctanassa violacea (L.) (Oiseau Ardéiforme).

Habitat: Gros intestin.

Localisation géogRAPHIQUe: Manguinhos (Rio).

DATE DE RÉCOLTE : 15.2.1912.

MATÉRIEL OBSERVÉ : La préparation contient un exemplaire du parasite (syntype) en orientation ventrale, modérément aplati à la fixation et en bon état de conservation.

Complément de descriplion (fig. 3).

Corps élancé de $800 \times 285 \mu$. Cuticule épineuse antérieurement jusqu'au niveau des testicules sur les $2 / 3$ antérieurs du corps (épines en forme d'écailles, de 4,5 $\times 2,5 \mu$ au niveau du pharynx). V.O. de $90 \mu \times 85 \mu$. V.V. de $80 \times 100 \mu$ post-équatoriale. Prépharynx de $60 \mu$. Pharynx de $45 \times 35 \mu$. Esophage de $150 \mu$. Caeca courts de 110 et $120 \mu \times 25-30 \mu$ de $\varnothing$. Pore génital senestre, situé ventralement et latéralement à $55 \mu$ du bord le plus proche de l'acátabulum. Atrium génital ventral très réduit. Poche du cirre de $150 \times 25 \mu$ de $\varnothing$ à paro:s minces $(1 \mu$. ne contenant qu'une petite vésicule séminale de $30 \times 25 \mu$. Canal éjaculateur large, glabre (?), rectiligne, long au total de $70 \times 13$ à $20 \mu$ de $\varnothing$. Ovaire dextre, lobé, situé en avant du testicule droit, partiellement engagé sous les follicules vitellins. Testicules symétriques, très latéraux, relativement très postérieurs, lobés, partiellement recouverts par les follicules vitellins, mais non recouverts par l'utérus. Vitellogènes linéaires formés de gros follicules globuleux, latéraux, s'étendant ventra!ement depuis le fond du caecum droit et la région post-métratermique à gauche, jusqu'au niveau du bord postérieur des deux testicules. Dix follicules environ à droite occupent une aire de $190 \times 65 \mu$, et huit follicules à gauche une aire de $135 \times$ $60 \mu$. Vitelloductes transverses pré-testiculaires et post-acétabulaires. Utérus post-acétabulaire découvrant entièrement les testicules. Eufs très abondants de 15 à $17 \mu$ de long. Métraterme musculeux long de $100 \mu$ environ cheminant au sein d'une masse cellulaire glandulaire en décrivant une boucle. Système excréteur non apparent, sauf une portion des canaux collecteurs principaux ventraux.

Discussion. - Nous nous proposons de préciser les espèces faisant partie du ger re Odhneria dans une note ultérieure. 


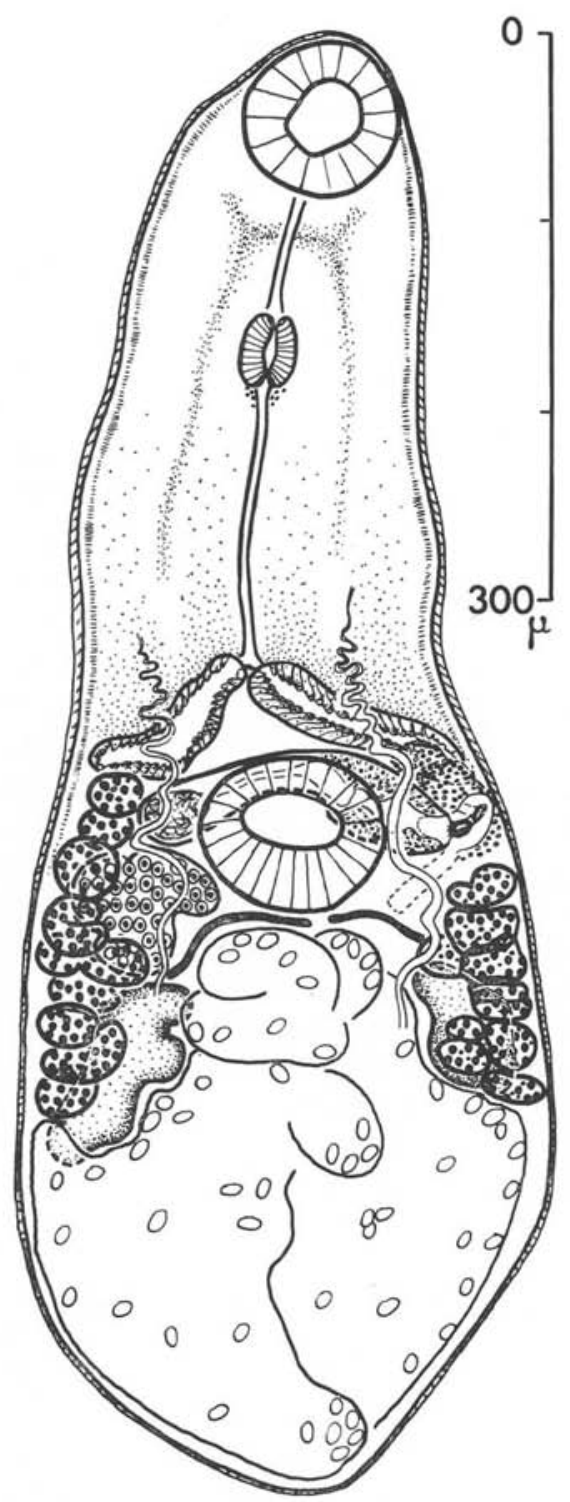

FIG. 3. - Odhneria odhneri Trav., 1921. Syntype. Vue ventrale 


\section{II. - GYNAECOTYLINAE}

Gynaecotyla jaegerskioeldi (Travassos, 1920)

syn. Levinseniella $j$. Travassos, 1920.

Préparation $\mathrm{n}^{\circ} 2.236$.

Hôte : Rallus cayennensis (Oiseau Ralliforme) [= Aramides cajanea (Müller)].

HaBITAT: Intestin.

Localisation GÉOgRAPHiQue : Manguinhos (Rio).

DATE DE RÉCOLTE : ?

MATÉRIEL OBSERVÉ : La préparation contient un exemplaire unique du parasite très aplati à la fixation, en position dorsale et parfaitement lisible. Il parait correspondre au trématode figuré de la planche XXIII de la description originale.

Complément de description (fig. 4).

Corps de $640 \times 400 \mu$ de large (très aplati). Cuticule épineuse sur les deux tiers antérieurs du corps jusqu'au niveau des testicules. Ventouse orale : $60 \mu$ de $\varnothing$. V.V. porale : $45 \mu$ de $\varnothing$. V.V. antiporale $56 \times 52 \mu$ (1). Prépharynx : $15 \mu$. Pharynx : $32 \times$ $32 \mu$. Esophage rectiligne: $113 \times 5-6 \mu \varnothing$. Caeca moyens de $230-240 \times 20$ $25 \mu$ dont les fonds atteignent le niveau du contour postérieur des acétabulums, en avant des testicules. Poche vésiculo-prostatique (P.V.-P.) : $170 \times 45 \mu$ à parois mus. culeuses épaisses de $2,5 \mu$. Vésicule séminale de $120 \times 32 \mu$. Cornucotyle (2) de $68 \times 80 \mu$ à l'état assez aplati. Trois faisceaux musculaires sous-tendent l'arc de la P.V.P. Testicules : $54 \times 80 \mu$. Ovaire senestre : $56 \times 80 \mu$. Utérus décrivant de nombreuses anses dans toute la partie du corps postérieure aux acétabulums et recouvrant les testicules. Métraterme peu différencié situé dorsalement entre l'acétabulum poral et la paroi gauche de l'atrium génital, long de $65 \mu$. Glandes vitellogènes en grappe de follicules nombreux situés latéralement et dorsalement en arrière des testicules. Vitelloductes tranverses post-testiculaires. CEufs de 17 à $19 \mu$. Pore génital non perceptible. Douze solénocytes localisables. Vessie non visible.

Discussion. - Les caractéristiques de l'espèce justifient l'attribution du distome au genre Gynaecotyla Yamaguti, 1939 proposée par Yamaguti lors de la création du genre, et simultanément et indépendamment par Rankin lorsqu'il décrivit Cornucopu'a en 1939.

En 1968, Deblock et Pearson proposaient une éventuelle synonymie de l'espèce avec G. adunca (Linton, 1905), après avoir considéré la grande similitude des mensurations des deux espèces du Nouveau-Monde. L'examen du syntype de Travassos confirme cette

(1) Dans le genre Gynaecotyla, il semble que la ventouse porale constitue l'acétabulum. La ventouse anti-porale, de par sa musculature originale, pourrait être considérée comme une néo-formation.

(2) Appareil copulateur hermaphrodite des genres Gynaecotyla et Diacetabulum. 


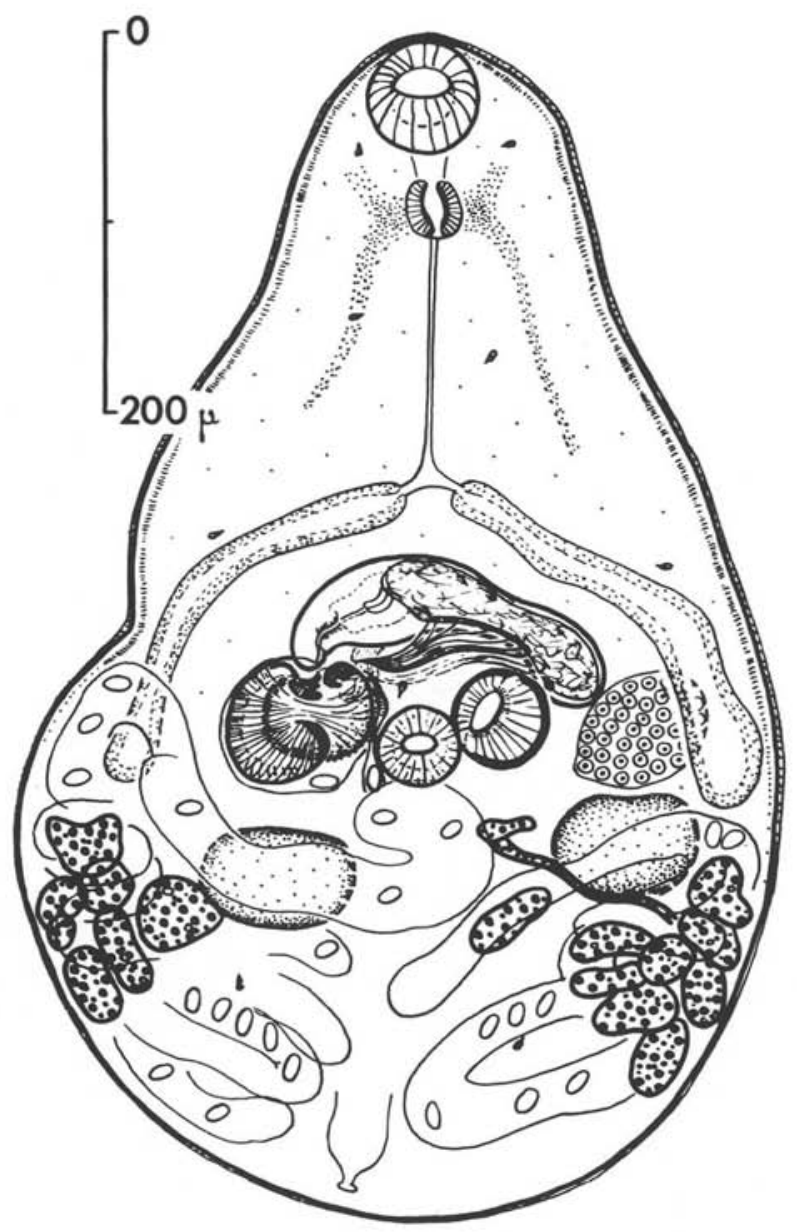

Fig. 4. - Gynaecotyla jaegerskioeldi (Trav., 1920). Syntype. Vue ventrale

synonymie ; l'examen comparé des cornucotyles (3) ne montre pas de différences fondamentales, bien que l'exemplaire de Travassos présente un moindre degré de sclérotisation de ses pièces constitutives.

(3) Notre matériel de référence en provenance des Etats-Unis d'Amérique est constitué par : 1. - le type de D. aduncum Linton, 1905, conservé à l'U.S.N.M.; il est en très mauvais état, « vitrifié » dans le baume, et ne fournit que des enseignements incomplets;

2. - plusieurs métacercaires dékystées de Uca pugilator du Massachusetts aimablement communiqués en 1961 par W. G. Hunter ;

3. - deux exemplaires adultes de Caroline du Nord fournis par R. Heard en 1967. Nous renouvelons nos remerciements à ces deux auteurs. 


\section{III. - MICROPHALLINAE}

$1^{\circ}$ Levinseniella cruzi Travassos, 1920.

PRÉPARATION n ${ }^{\circ} 2.079$ (comme $M$. nicolli; cf. supra).

MATÉRIEL OBSERVÉ: La préparation contient un exemplaire unique du parasite modérément aplati à la fixation, en orientation ventrale. Il est mal lisible.

Complément de description (fig. 5)

Corps de $600 \times 330 \mu$. Cuticule épineuse antérieurement jusqu'au niveau des testicules (épines de $8 \mu$ au niveau du pharynx). V.O. de $105 \times 108 \mu$ sans auricule. Sphincter pré-pharyngien non perceptible si présent. V.V. de $75 \times 55 \mu$. Tube digestif

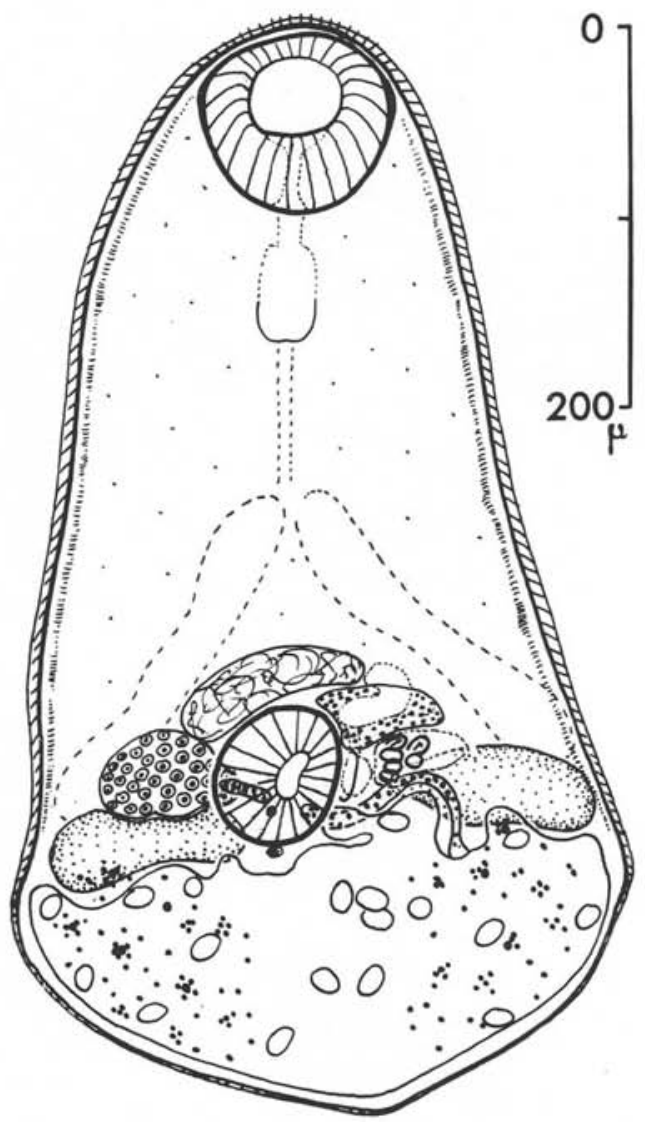

Fig. 5. - Levinseniella cruzi Trav., 1920. Syntype. Vue ventrale 
non discernable. Testicules de $50 \times 105 \mu$. Vésicule séminale de $80 \times 40 \mu$. Pars prostatica et papille mâle non discernables. Ovairz dextre de $50 \times 73 \mu$. Utérus ne dépassant pas antérieurement le niveau des testicules. Eufs abondants de 19 à $20 \mu$ de long. Métraterme non perceptible. Glandes vitellogènes diffuses post-testiculaires. Atrium génital à peine observable: poche femelle probablement absente; poches mâles peut-être au nombre de six, mesurant $13 \times 10 \mu$.

Discussion. - Il s'agit d'un Levinseniella typique, hypothétiquement du sousgenre Monarrhenos Dzblock et Pearson, 1970, dont la description originale ne peut être utilement complétée du fait d'une trop mauvaise conservation.

\section{$2^{\circ}$ Apophallus simillimus Travassos, 1920.}

PréPARATION $\mathrm{n}^{\circ} 2.116$.

Hôte: Nyctanassa violacea (L.) (Oiseau Ardéiforme).

HABITAT: Intestin grêle.

Localisation gÉOgRAPHIQUe: Manguinhos (Rio).

DATE DE RÉCOLTE : ?-9-1920.

La préparation contient un exemplaire unique (cotype) du parasite relativement bien conservé, mais de lecture difficile, l'atrium génital étant obscurci par la présence sous-jacente de l'utérus empli d'œufs. Il se situe en orientation dorsale. Il ne paraît pas correspondre exactement à l'exemplaire figuré pl. XXIV de la description originale.

\section{Complément de description (fig. 6).}

Corps de $440 \times 250 \mu$. Cuticule couverte d'épines de 4 à $5 \mu$ de long au niveau du pharynx et très atténuées à partir du niveau des testicules. V.O. : $62 \times 65 \mu$. Pharynx $25 \times 17 \mu$. Esophage $113 \mu$. Caeca $113 \times 17 \mu$. V.V. $55 \times 62 \mu$. Ovaire dextre $40 \times 56 \mu$. Testicules peu visibles. Vésicule séminale pré-acétabulaire peu discernable (28 $\times 56 \mu$ ?). Utérus empli d'œufs de 17-19 $\mu$ ne dépassant pas le niveau du caeca, mais recouvrant les testicules. Vitellogènes diffus situés ventralement au niveau des testicu'es. Papille mâle musculeuse apparaissant comme un anneau charnu de $18 \mu$ de $\varnothing$ environ, situé du côté gauche de l'acétabulum.

Commentaire. - Sur ce distome à morphologie de Microphallus classique, il n'est pas possible de discerner les formations anatomiques musculeuses évoquées p. 89 dans la description originale et que le dessin XXIV esquisse. Le pore génital ventousiforme paraît correspondre à ce que l'on peut interpréter comme une papille mâle de Microphallus, d'un type sphéroïdal banal. Selon nous, il ne s'agit ni d'une espèce du genre Levinseniella ni d'un Gynaecotylinae.

Il n'y a pas de poche du cirre transversale de 106 à $113 \mu$. 


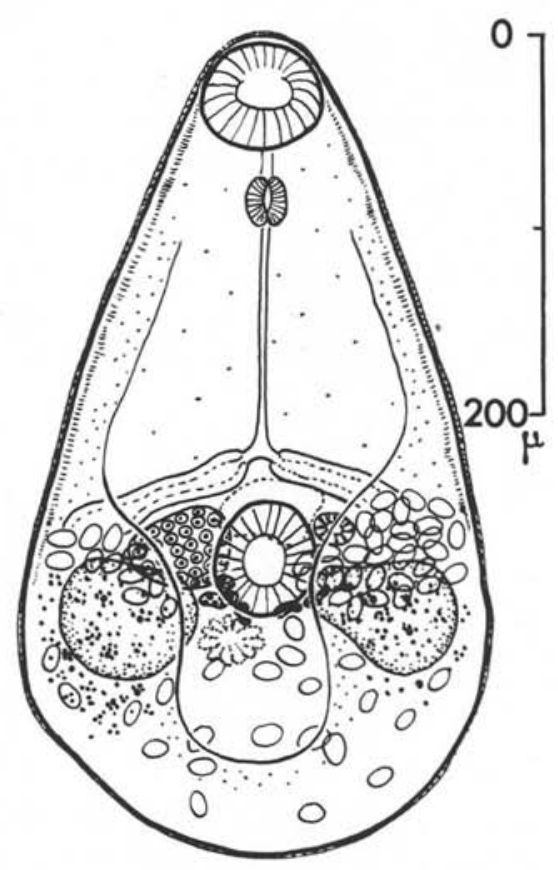

FIG. 6. - Apophallus simillimus Trav., 1920. Syntype. Vue ventrale

Remarque. La préparation $\mathrm{n}^{\circ} 2.067$ [contenu du gros intestin de Dafila bahamensis, autopsié le 23-2-1912 à Manguinhos (Rio)], présente un unique exemplaire d'un Microphallidé de $200 \mu$ de long que nous identifions à Atriophallophorus minutus (Price, 1934). Cela étend à l'Amérique du Sud la répartition géographique de ce Microphallidé déjà signalé en Amérique Centrale, en Amérique du Nord et en Europe.

Il ne paraît pas que Travassos l'ait décrit ni dénommé.

\section{Bibliographie}

Belopolskaia (M. M.), 1952 et 1963. - La famille des Microphallidae Travassos, 1920, in K. I. Srkiabine, Trématodes des animaux et de l'homme, 1952, 6, p. 619-756 et 1963,21 , p. 259-504 (en russe).

DeBlock (S.), 1972. - Contribution à l'étude des Microphallidae Travassos, 1920, XXIV. Tentative de phylogénie et de taxonomie. Bull. Mus. Nat. Hist. Nat. (Paris), $3^{\mathrm{e}}$ sér., Zoo., 7, 110 p. (sous presse). 
Travassos (L.), 1920. - Contribuição para conhecimento da fauna helmintologica brasileira - IX. Sobre as especies da subfamilia Microphalinae Ward, 1901. Arch. Escola Super. Agric. Med. Veter., Nictheroy, 4 (2), p. 85-91.

—, 1921. - Contribuiçoes para o conhecimento da fauna helmintholojica brasileira. XII. Sobre as especies brasileiras da subfamilia Brachycaelinae. Arch. Escola Super. Agric. Med. Veter., Nictheroy, 5 (1-2), p. 59-67. Pl. IX-XI.

-, 1929. - Une nouvelle espèce du genre Maritrema, Maritrema pulcherrima n. sp., Trematoda. C.R. Soc. Biol. Paris, 100, p. 945-946. 